\title{
A DELICADA TAREFA DE AVALIAR A APRENDIZAGEM NA FORMAÇÃO POR COMPETÊNCIAS EM STEM
}

DOI: 10.37702/2175-957X.COBENGE.2021.3710

Laurete Zanol Sauer - Izsauer@ucs.br

UNIVERSIDADE DE CAXIAS DO SUL

Rua Tarquinio Zambelli 164

95054-410 - Caxias do Sul - RS

Isolda Gianni de Lima - iglima1@gmail.com

Universidade de Caxias do Sul

Rua Guia Lopes, 670, apto 51670

95020-390 - Caxias do Sul - RS

Derlise Fiametti Xavier - dfxavier1@ucs.br

Prefeitura Municipal de Farroupilha

Rua Cavalheiro Horácio Mônaco 104

95700-050 - Bento Gonçalves - RS

Diana Isabel de Araújo Mesquita - diana@dps.uminho.pt

Universidade do Minho

Rua António Fiuza de Melo 101

-_ _ Vila Nova de Famalicão -

Dianne Magalhães Viana - diannemv@unb.br

UnB

SQSW 304 Bloco C Apto 607 Bloco C Ap

70673-403 - Brasília - DF

Odilon Giovannini - ogiovannini@gmail.com

Universidade de Caxias do Sul

R. Aguinaldo da Silva Leal 45

95700-358 - Bento Gonçalves - RS

Valquíria Villas-Boas - vvillasboas@gmail.com

Universidade de Caxias do Sul

Rua Francisco Getúlio Vargas 1130

95070-560 - CAXIAS DO SUL - RS 
Resumo: Neste trabalho, tomamos como ponto de partida a eficácia, já comprovada, da utilização de estratégias e métodos de aprendizagem ativa na área de STEM. Com efeito, entendemos, que se trata de uma alternativa metodológica para dar conta, não somente da superação de dificuldades relacionadas à aprendizagem, mas também para atender às expectativas quanto ao perfil e às competências que nossos estudantes precisam desenvolver. Diante dessas considerações, justificamos nossas concepções sobre os processos de ensino, de aprendizagem e de avaliação e apresentamos algumas possibilidades já identificadas, de estratégias de aprendizagem ativa e de avaliação, passíveis de promoverem o desenvolvimento de competências, no contexto das novas Diretrizes Curriculares Nacionais para os cursos de graduação em Engenharia.

Palavras-chave: Avaliação, Competências, STEM, Educação em engenharia, DCNs 


\section{A DELICADA TAREFA DE AVALIAR A APRENDIZAGEM NA FORMAÇÃO POR COMPETÊNCIAS EM STEM}

\section{INTRODUÇÃO}

Ao longo das últimas décadas, diversos países passaram a preocupar-se não apenas com a disponibilização de acesso e com a frequência dos estudantes às instituições de ensino formal, mas também com a qualidade do ensino ofertado na Educação Básica. Preparar esses estudantes para a universidade e seus desafios, para o mercado de trabalho onde o conhecimento é alicerce para tomada de decisões e, principalmente, para o pleno exercício da cidadania, que propicia o reconhecimento da pessoa como sujeito ativo dentro de sua realidade nacional, passaram a ser objetivos das nações que almejam a prosperidade de seu povo.

Nasceram, assim, mecanismos que buscam orientar os países a respeito da qualidade da Educação Básica, que conjuntamente a outras informações de seus estudantes, possibilitam a avaliação, a construção e a redefinição de políticas públicas educacionais estratégicas para seu desenvolvimento, além de proporcionar informações para a sociedade.

Dentre os mecanismos citados, destacam-se as avaliações externas, tais como o Programa Internacional de Avaliação de Estudantes - PISA e o Estudo Regional Comparativo e Explicativo - ERCE, nos quais nações firmam coligação internacional de esforços avaliativos de seus estudantes, e o Sistema de Avaliação da Educação Básica SAEB, este nacional, que trazem dados informativos sobre os níveis de aprendizagem dos estudantes e, consequentemente, da qualidade dos sistemas de ensino.

Deve-se, diante do quadro acima delineado, dentro de um mundo globalizado, onde a informação é passível de comparação sob diversos enfoques, inclusive seu ordenamento na forma de ranking, atentar aos resultados das avaliações em larga escala recentemente obtidos pelo país, estado e pelo município, nos níveis internacional e nacional, assim como seus reflexos, que geram inquietação na comunidade, na imprensa e também constantes reflexões da academia, estas geralmente negativas (DE LIMA et al., 2020), quanto à prática metodológica docente e à qualidade do ensino ofertado.

No atual contexto internacional, em que a educação brasileira é posta à prova e é apurado que 68,1\% de nossos alunos que cursavam o 9 ano do Ensino Fundamental foram classificados no PISA 2018 no pior nível de proficiência em Matemática, sendo que destes, $41 \%$ não puderam ter habilidades avaliadas pelo programa em razão de não atingirem escore mínimo na avaliação (INEP, 2019, p.110), ações têm de ser tomadas para reverter esta situação.

É passado da hora para que não só reflitamos sobre práticas pedagógicas no âmbito acadêmico, mas que sejam elas aplicadas à realidade de nossas crianças e jovens, com vistas a conquistarem a tão propalada dignidade da pessoa humana, prevista no Art. $1^{\circ}$, III da Constituição Federal de 1988. Segundo a Ministra do Supremo Tribunal Federal, Cármen Lúcia Antunes Rocha, "A garantia da dignidade da pessoa humana é o princípio mais importante na Constituição de 1988" e destaca ainda que "A Constituição criou sistemas, como o de educação, que é libertadora" (BRASIL, 2018), pensamento há muito defendido por Paulo Freire (FREIRE, 1967).

O documento político-jurídico ainda prevê no Art. 205 que "A educação, direito de todos e dever do Estado e da família" e que a educação visa "ao pleno desenvolvimento da pessoa, seu preparo para o exercício da cidadania e sua qualificação para o trabalho". Ora, não são outros os objetivos visados pelo conceito de letramento em Matemática definido 
pela OCDE em 2010 (INEP, 2010), com pequenas modificações no passar dos anos, quando afirmava que o objetivo era o de "atender as necessidades do indivíduo no cumprimento de seu papel de cidadão consciente, crítico e construtivo". O não letramento matemático dos estudantes é um fator que contribui para a não escolha por cursos nas áreas de Ciências Exatas, Matemática, Tecnologias e Engenharias (STEM), ou caso a escolha seja feita, pelas inúmeras dificuldades que os estudantes encontram para terem um bom desempenho nestes cursos.

Assim, pensar a avaliação tanto na sala de aula quanto em larga escala é fundamental para o futuro da área de STEM. No Ensino Superior, os estudantes continuarão a ser avaliados em seus cursos e em avaliações externas. Se esses estudantes forem expostos a processos de ensino e de aprendizagem, nos quais os resultados de aprendizagem pretendidos, a metodologia de desenvolvimento das aulas e os instrumentos de avaliação estejam fundamentados em uma concepção epistemológica construtivista, as chances de se formar profissionais competentes e alinhados com o seu tempo aumentam consideravelmente.

As exigências em relação à formação de cientistas e engenheiros na contemporaneidade fazem emergir a necessidade do desenvolvimento de competências relacionadas à conquista da autonomia intelectual, à capacidade de aprender sozinho, de aprender a aprender e de lidar com problemas, sendo criativo e inovador, dentre outras (BRASIL, 2019).

Para tanto, estudos indicam que os métodos e as estratégias de aprendizagem ativa, focados nas ações intelectuais do estudante, em convivência com seus colegas de estudo, com base nas orientações do professor, são uma alternativa a ser considerada nos planejamentos de cursos, de disciplinas e de aulas. Tais métodos e estratégias, quando baseadas no fazer e compreender, podem promover a tomada de consciência necessária, imprescindível à aprendizagem. Por exemplo, no caso de aprendizagem de Matemática, isto significa compreender as ideias que perpassam definições, regras, fórmulas e procedimentos algorítmicos; saber utilizar as várias formas de expressão matemática: algébrica, geométrica, numérica e verbal, na organização de textos próprios, o que implica saber ler e interpretar corretamente textos matemáticos, produzindo justificativas e argumentações coerentes para os procedimentos adotados na resolução de problemas (SOARES, LIMA, SAUER, 2008). No caso de aprendizagem em Física, isto significa compreender tudo o que foi exposto para a Matemática e a isso agregar a compreensão dos fenômenos físicos e, no caso das Tecnologias e da Engenharia a compreensão de contextos complexos, dos avanços da ciência e da tecnologia, bem como em relação aos desafios da inovação. Para tanto, ambientes de aprendizagem ativa, centrados em estratégias e intervenções baseadas em interações sociocognitivas, podem levar à construção de conhecimento por meio de reflexões, desencadeadas por discussões e trocas de ideias, fundamentadas em argumentos e justificativas, baseadas na teoria estudada, onde a prática mecânica de exercícios é substituída por um cenário de investigação, onde os estudantes são convidados a se envolverem em processos de exploração e de argumentação justificada, assumindo sua parcela de responsabilidade nos respectivos processos de aprendizagem. Conforme Lima (2004), um ambiente de aprendizagem de Matemática deve promover situações que levem os estudantes a produzirem significados e a compreenderem conceitos quando realizam atividades matemáticas. Sauer (2004) destaca a importância do diálogo matemático que pode surgir do processo de construção de significados e de argumentação em torno das tarefas propostas, envolvendo a resolução de problemas. Essa forma de planejar o processo de aprendizagem, conforme Soares (1997), desloca o foco do conteúdo programático para o desenvolvimento de competências cognitivas, no âmbito da Matemática, entendidas como 
operações realizadas para estabelecer relações entre os objetos, situações, fenômenos e conceitos matemáticos. As mesmas ideias podem ser estendidas à Física e à Engenharia. Assim, nesse sentido, a aprendizagem das disciplinas da área de STEM pode capacitar os estudantes a entender estas disciplinas, não apenas em sua dimensão formal, mas também, e especialmente, em sua dimensão formadora de condutas requeridas para os cientistas e engenheiros, de acordo com as novas Diretrizes Curriculares Nacionais para os cursos de graduação em Engenharia (DCNs) (LIMA, SAUER, SOARES, 2007).

Essa forma de entender e de planejar o processo pedagógico no contexto da aprendizagem de STEM, pode ser capaz de desenvolver as habilidades e competências, apresentadas nos planos dos cursos: ser criativo, questionador, apresentar atitude científica (observar, identificar variáveis intervenientes, elaborar e testar hipóteses, analisar dados e informações, propor metodologias de investigação que incluam abordagem da complexidade), gerenciar o tempo (classificar tarefas por prioridade, importância e urgência), comunicar-se com argumentações, fatos, dados de forma esclarecedora; realizar pesquisas bibliográficas, buscando dados e informações, gerenciar conhecimento, resolver problemas, conhecer técnicas ou propor técnicas de resolução de problemas: conjunto de procedimentos que levem à solução de situações-problema (LIMA, SAUER, SOARES, 2007).

Dessa forma, para aprender na interação em classe faz-se necessário dialogar, interagir, questionar e problematizar para possibilitar a superação de dificuldades, em um processo que envolve diferentes linguagens. Em STEM, a linguagem escrita e falada, com reconhecimento e uso adequado da simbologia matemática, acrescenta conhecimentos novos aos já desenvolvidos, unindo-os na construção de novos conceitos.

Aos processos de ensino e de aprendizagem junta-se a avaliação, que os integra e complementa, fornecendo um diagnóstico que permite repensar e reformular métodos, procedimentos e estratégias e métodos de aprendizagem (VILLAS BOAS et al., 2012; ELMÔR-FILHO et al., 2019).

Este artigo está organizado em quatro seções, a saber: introdução, avaliação formativa integrando a aprendizagem por competências, estratégias de aprendizagem ativa como instrumentos da avaliação formativa e as considerações finais.

\section{AVALIAÇÃO FORMATIVA INTEGRANDO A APRENDIZAGEM POR COMPETÊNCIAS: ASPECTOS PEDAGÓGICOS E AS NOVAS DCNS}

Partimos da concepção de avaliação, como um processo formativo, mapeando e compreendendo como está acontecendo a aprendizagem: quais as dificuldades, quais os obstáculos, quais os avanços, que aspectos precisam ser aperfeiçoados, considerando também as emoções que perpassam esse contexto: ansiedades, medos, receios.

Assim concebida, a avaliação fornece dados e informações para que o professor programe intervenções pedagógicas, dicas e orientações, problemas e desafios para que os estudantes estabeleçam relações e desenvolvam habilidades e condutas de valor. Dessa maneira, a avaliação não é apenas um momento específico, mas integra o processo pedagógico de forma contínua, constituindo-se em um processo contínuo de diagnosticar dificuldades e obstáculos de aprendizagem, como fonte de reinvenção da prática pedagógica (LIMA, SAUER, SOARES, 2007).

De acordo com Cid (2017), a avaliação, vista como um veículo de melhoria das aprendizagens, traz em si uma aposta na criação de condições efetivas para a aprendizagem de todos, promovendo uma cultura de sucesso, apoiada no pressuposto de que todos podem aprender. Esta perspectiva configura procedimentos de melhoria dos processos de ensino e de aprendizagem, recriação do currículo e implica incrementar 
contextos avaliativos que se contraponham a uma racionalidade técnica, tradicional, e que apostem na avaliação como prática reflexiva da ação, prevenindo a segregação e valorizando a aprendizagem. Quando a avaliação é considerada como um modo de adequação das estratégias e dos métodos pedagógicos focados nas especificidades dos estudantes, cria as condições necessárias para se tornar um veículo de inclusão educacional.

A avaliação é por si só um conceito complexo e inclui uma grande carga polissêmica. Por avaliação poderemos ter diferentes concepções e o que realmente importa é que cada docente seja capaz de identificar qual é a sua concepção com base na sua prática - diz-me como avalias e dir-te-ei quem és (GUERRA, 2003). Os propósitos da avaliação vão além da avaliação da aprendizagem - uma avaliação certificadora e somativa - principalmente, quando falamos de um currículo por competências. Assim importa considerar duas outras funções da avaliação (HADJI, 1994), nomeadamente: avaliação para a aprendizagem: uma avaliação formativa, centrada em dinâmicas de feedback ao longo do processo permitindo que o estudante seja capaz de regular a sua aprendizagem ("Onde, como e quando posso melhorar o meu desempenho?"); e avaliação como aprendizagem - em que o estudante participa no processo de avaliação, a partir, por exemplo, de modelos de avaliação pelos pares.

Nesse sentido, avaliar competências pressupõe integrar as diferentes funções inerentes à avaliação (da / para / como), diversificando os instrumentos a serem utilizados, com vista a avaliar diferentes níveis de competências.

\section{ESTRATÉGIAS DE APRENDIZAGEM ATIVA COMO INSTRUMENTOS DA AVALIAÇÃO FORMATIVA CONTEMPLANDO AS NOVAS DCNs}

O que apresentamos a seguir revela nossa concepção de avaliação integrando o processo pedagógico, como base para o professor criar suas intervenções e estratégias e para o estudante repensar sua forma de aprender e de participar ativamente, através de ações de autorregulação e de metacognição. Trata-se de uma seleção de instrumentos de avaliação que temos utilizado, entendendo-os como possibilidades de dar conta dessas concepções de ensinar com base no desenvolvimento de competências, de aprender com base na compreensão e de avaliar com base no autoconhecimento e na tomada de consciência.

$\mathrm{O}$ ato de aprender distingue a aprendizagem como ativa e significativa, que, segundo Ausubel (2003), produz um novo conhecimento ancorado na estrutura de conhecimentos já adquiridos e que resulta de um processo no qual o professor cria condições para 0 estudante interagir.

Para Moraes (2007), os conceitos nunca estão prontos, pois sempre podem ser enriquecidos e complexificados. Conhecimentos novos são aprendidos por reconstrução constante daqueles já anteriormente construídos, o que requer interagir com algo já conhecido, e transformá-lo, tornando-o mais complexo e amplo.

De acordo com esta concepção, ensinar deve ser, então, favorecer as ações cognitivas, propiciadas por meio de atividades, orientações e intervenções pedagógicas que auxiliem o estudante a realizar suas tarefas, e aprender com elas, a elaborar ideias próprias e modificá-las à medida que amplia o seu conhecimento (SAUER, LIMA, SOARES, 2008).

A interação entre os estudantes promove a cooperação, com o sentido de atuar, operar com o outro, compartilhando ideias, significados e conhecimentos, seus e do outro, modificando ambos. Um processo de interação e cooperação traz consigo o diálogo, que valoriza todos os tipos de saberes e, também, o saber do outro. 
Diante dessas considerações, selecionamos e passamos a descrever algumas estratégias que temos utilizado, em que entendemos estar promovendo aprendizagem ativa e a avaliação da aprendizagem, ao mesmo tempo, com potencial para o desenvolvimento de competências que possam ser avaliadas.

Estas estratégias podem ser utilizadas como instrumentos de avaliação de disciplinas da área de STEM e podem ser aplicadas tanto em ambiente presencial como em ambientes online. São elas:

\subsection{Elaboração de resumos}

Solicitar que os estudantes sistematizem suas ideias acerca do entendimento de um dado conceito, com base em pesquisas bibliográficas e nas explicações do professor, elaborando com suas palavras o significado e as possibilidades de aplicação do conteúdo que está sendo estudado, sejam eles teoremas, fórmulas ou regras, na resolução de situações-problema. Tais sistematizações resultam em textos que podem ser objeto de consulta no decorrer de provas escritas. Essa estratégia auxilia no desenvolvimento da capacidade de elaborar textos e de comunicar suas ideias de forma clara e organizada.

\subsection{Identificação e reconhecimento de dificuldades}

Solicitar que os estudantes identifiquem e destaquem dificuldades, bloqueios, angústias, variáveis que estão interferindo, sejam elas de ordem emocional, conceitual ou cognitiva, sejam de ordem lógica, de pensamento ou de pré-requisitos. Essa estratégia de análise, realizada pelos estudantes com auxílio do professor, pode proporcionar o desenvolvimento de habilidades de metacognição e de autoconhecimento, que podem levar ao desenvolvimento de competências de natureza psicossocial (ser e estar): relacionar-se com os sentimentos e as emoções, pensar em alternativas para superação, reconhecendo suas qualidades e, também, erros e fraquezas. Podemos incentivá-los a construir um projeto de vida e de aprendizagem.

\subsection{Atividades de autoavaliação}

Essa estratégia está baseada na concepção de autoavaliação entendida como um mecanismo de autorregulação do processo de aprendizagem, pois pode auxiliar o estudante a entender como está acontecendo a sua aprendizagem. Para Coll (1994), a autorregulação da aprendizagem implica a competência de realizar aprendizagens significativas por si só, em uma ampla gama de situações e circunstâncias. E, regulação dos processos de aprendizagem, conforme Perrenoud (1999), é o conjunto das operações metacognitivas do sujeito e de suas interações com o meio que modificam seus processos de aprendizagem no sentido de um objetivo definido de domínio. Sob esses pontos de vista, uma função importante da autoavaliação é indicar ao estudante (localizar, explicitar) o que precisa ser feito, revisto, estudado, (re)elaborado para superar dificuldades e para o desenvolvimento de estruturas cognitivas. Atividades de autoavaliação podem, então, fornecer ao professor, indicativos de estratégias e intervenções pedagógicas capazes de auxiliar no desenvolvimento da autonomia.

\subsection{Análise de erros}

Solicitar que os estudantes analisem os erros de uma avaliação escrita, identificando os motivos e os procedimentos incorretos, buscando na teoria argumentos e justificativas. Ao analisar e discutir com os estudantes, os procedimentos adotados em resoluções de problemas, o professor pode utilizar os erros ou acertos como fontes de reconhecimento do que precisa ser (re)elaborado para que sejam superadas as dificuldades (LIMA; SAUER, 2002). Conforme Piaget (1978), um erro pode ser mais fecundo do que um acerto imediato, 
porque a comparação entre uma hipótese falsa e suas consequências fornece novos conhecimentos e a comparação entre dois erros pode levar a elaboração de novas ideias.

\subsection{Avaliação da avaliação/prova}

A "avaliação" da avaliação é realizada por professor e estudantes na aula seguinte à realização de uma avaliação parcial. Ela não consiste exatamente na resolução completa dos problemas da avaliação pelo professor, mas de um momento de questionamento sobre os conhecimentos abordados na avaliação e das dificuldades que o aluno teve em demonstrar a construção e o estabelecimento de relações deste conhecimento. A etapa de repensar e refazer a avaliação é o passaporte para que o estudante possa fazer uma prova substitutiva. Acreditamos que ao refazer a avaliação o aluno tem mais um momento em que pode exercitar os conceitos que deveria ter construído e/ou que construiu.

\subsection{Discussões coletivas}

Usar o fórum de um ambiente virtual como local para discussões em torno de tarefas iniciadas em encontros presenciais ou como atividades que complementem esses encontros. Essa estratégia pode abrir espaços para a construção de novos saberes. Nela estudantes e professores, por meio de ações problematizadoras e sistematizadoras, podem observar, questionar, comentar e dar dicas, promovendo reflexão crítica e elaboração de novas ideias. Para que isso aconteça, as tarefas de aprendizagem devem ser elaboradas de forma a promover a reflexão, a argumentação, a interpretação e a dedução, e não apenas cálculos mecânicos repetitivos e desprovidos de significado.

* Estudos complementares de temas iniciados em sala de aula.

* Estudos de introdução a novos temas, depois complementados e continuados em aulas presenciais.

* Tarefas de estudo com vistas à avaliação (individuais e ou em grupos) tais como: resolução de problemas, pré-provas, complemento e aperfeiçoamento de provas, sempre de forma colaborativa.

* Discussões como forma de compartilhar conhecimentos por meio da resolução colaborativa de tarefas, aperfeiçoamento de provas ou esclarecimento de dúvidas.

\subsection{Texto colaborativo}

A elaboração de textos colaborativos é uma outra estratégia de avaliação bastante poderosa. Por meio de um editor colaborativo, como o do Google Drive, os estudantes produzem seus trabalhos na forma escrita, usando cores distintas. A construção de um texto colaborativo pode ser um grande desafio para os estudantes, mas também pode ser muito recompensador. Este texto pode ser um relatório de laboratório, um relatório de trabalho relacionado a um problema ou projeto, uma análise de uma palestra, uma resenha de livro, entre outros.

\subsection{Proposição de desafios para serem resolvidos através de discussões compartilhadas}

Nesse caso, todos podem solicitar ou fornecer explicações sobre os passos desenvolvidos ou os recursos utilizados, argumentando com base nos estudos e na teoria. Essa estratégia auxilia a mostrar que não existe um único caminho para resolver determinado problema, como é usual pensar no caso da Matemática. Também auxilia o estudante a decidir se um determinado processo de resolução está ou não adequado, bem como se o resultado obtido está correto. As interações são, portanto, alimentadas por discussões dos interagentes, professor e estudantes, exigindo esforços na compreensão, no fazer-se entender pelo outro e na clareza na escrita, que é a base da comunicação nesse 
contexto. Essa estratégia tem produzido resultados bastante interessantes, especialmente em ambientes virtuais. Para tanto, tais ambientes, com seus espaços de aprendizagem, devem ser organizados com o propósito de, além de fornecer informações relacionadas aos conteúdos, acompanhar os estudantes com intervenções e orientações, mediadas por ferramentas de comunicação, que visam ao desenvolvimento da autonomia, da capacidade de lidar com problemas e com a tecnologia, e de tomar decisões com conhecimento e confiança. Além disto, o suporte tecnológico é o que permite a socialização das atividades desenvolvidas, suscitando o desenvolvimento da capacidade de entender o outro, de cooperar e de atuar em equipe. A distância física não impede a constituição de diálogos, cujos registros possibilitam, ao professor, condições de avaliar a aprendizagem, retomando os processos de pensamento em vários momentos, propondo novos desafios, sempre que oportuno, possibilitando a coconstrução, a coautoria e o registro dinâmico, podendo ser modificado, acrescentado ou transformado, o que se constitui em fonte de avaliação, aperfeiçoamento e desenvolvimento contínuos

\subsection{Circuito de integrais e derivadas}

Pode ser aplicado em diferentes situações. Na forma como tem sido aplicado, quando do estudo de integração, na disciplina de Cálculo Diferencial e Integral, tem-se a oportunidade de retomar as regras de derivação, já estudadas. Trata-se de uma interação em equipes para o conhecimento e aplicação de integrais e derivadas, com discussão sobre os procedimentos aritméticos, algébricos e sobre comunicação matemática adequada, que requer o formalismo da sua linguagem própria como expressão das ideias e dos significados que se deseja transmitir. $\mathrm{Na}$ aula anterior à do circuito, os estudantes são comunicados sobre a atividade de aprendizagem e de avaliação, como um convite a participarem de um desafio.

O lúdico como recurso pedagógico tem o poder de melhorar a autoestima dos estudantes e promover um ambiente descontraído e envolvente, servindo como estímulo para a interação, pois gera interesse e prazer em relacionar-se com os colegas. Libertos de uma situação mais formal de aprendizagem, e contando com o apoio dos colegas, todos se sentem mais à vontade para discutir, dizer que não sabem e o que não sabem, para pedir e dar palpites, sugestões e ideias. Desta forma, atividades competitivas, realizadas em equipes, em que estudantes resolvem problemas com auxílio de colegas, podem indicar o que o estudante pode fazer melhor do que conseguiria fazer por si mesmo. E, ainda, abrese a oportunidade de que amanhã consiga fazer sozinho aquilo que hoje é capaz de fazer em cooperação. Em qualquer dessas formas, o feedback é imprescindível, se o interesse for relacionar a avaliação com a aprendizagem. Nesse aspecto, ganha destaque a presença do professor, que não precisa ser aquele que responde todas as questões, que está sempre disponível, mas que é o centralizador de questionamentos que levem a discussões esclarecedoras, atitudes de encorajamento, de distribuição de tarefas e de auxílio entre os colegas.

\subsection{Minute Paper}

Nessa estratégia de aprendizagem ativa e de avaliação da aprendizagem, o professor finaliza a aula uns dois a cinco minutos antes de terminar o tempo previsto e pede aos estudantes que individualmente escrevam sobre o tema abordado na aula, solicitando que respondam questões como as que são apresentadas a seguir (SCHWARTZ, 1991; ANGELO; CROSS, 1993; ELMÔR-FILHO et al, 2019):

- Quais foram o(s) ponto(s) principal(is) da aula?

- Quais foram o(s) ponto(s) menos claro(s) da aula?

- Qual foi o conceito mais importante que aprendeu durante a aula? 
- Qual(is) a(s) pergunta(s) que gostaria de fazer?

- Qual o exemplo mais significativo, a imagem mais impactante, a informação mais surpreendente, a ideia mais perturbadora?

- O que você pode mudar na maneira como abordou [o assunto X, o problema $\mathrm{Y}, \mathrm{o}$ caso Z] na próxima vez que tiver uma tarefa semelhante?

- Explique sua experiência ao completar [o projeto X] e o que você aprendeu sobre como lidar com projetos como este.

Estas questões podem ser respondidas em um pequeno pedaço de papel, no Google Forms, ou em outro aplicativo ou espaço de registro que o professor achar mais conveniente. Uma vez respondida a questão, o professor explica que no próximo encontro, a aula será iniciada abordando questões comuns que foram apresentadas nos "relatórios" produzidos pelos estudantes, visando ao feedback necessário para a continuação dos estudos.

\subsection{Avaliação pelos pares}

A avaliação pelos pares é uma estratégia de avaliação formativa e pode ser entendida como um mecanismo de autorregulação do processo de aprendizagem. Estudantes fazem julgamentos sobre os trabalhos de outros estudantes, usando seus próprios critérios de avaliação ou os que são fornecidos pelos professores. Este tipo de avaliação é muito adequado para ser utilizado em associação com métodos colaborativos de aprendizagem ativa, tais como: Casos de Ensino; Problem-based Learning (Aprendizagem baseada em Problemas); Project-based Learning (Aprendizagem baseada em Projetos); Scenario-based Learning (Aprendizagem baseada em Cenários); Servicebased Learning (Aprendizagem baseada em Serviços Comunitários); Design Thinking; entre outros.

\subsection{Avaliação Colaborativa}

Avaliação Colaborativa é aquela na qual o estudante se avalia, à luz de critérios acordados com o professor. O professor avalia o estudante usando os mesmos critérios e negociam uma nota final. Como no caso da avaliação pelos pares, a avaliação colaborativa também é muito adequada para ser utilizada em associação com métodos colaborativos de aprendizagem ativa, já citados anteriormente.

No Quadro 1, apresentamos um conjunto de instrumentos de avaliação que também podem ser usados tanto em contextos presenciais quanto online.

Quadro 1 - Visão geral de instrumentos de avaliação.

\begin{tabular}{|c|c|c|c|}
\hline Testes/Exercícios & Produção de texto & $\begin{array}{c}\text { Desenvolvimento de } \\
\text { projeto }\end{array}$ & Comunicação oral \\
\hline $\begin{array}{c}\text { Teste em forma de } \\
\text { "prova" }\end{array}$ & Resumo/Resenha & Pôster & Seminário \\
\hline $\begin{array}{c}\text { Teste em forma de } \\
\text { "exercícios" }\end{array}$ & Artigo & Mapa conceitual & Apresentação de pôster \\
\hline Caso de Ensino & Relatório & Linha do tempo & Debate \\
\hline Situação-problema & Memorial & Vídeo/Podcast & Júri simulado \\
\hline & Ensaio & Software/Hardware & "Prova" oral \\
\hline & Postagem em fórum & Infográfico & \\
\hline & Portfólio & Maquete & \\
\hline & & Protótipo & \\
\hline
\end{tabular}

Fonte: Adaptado de documento produzido pelo Núcleo de Formação de Professores do Centro de Inovação e de Tecnologias Educacionais da Universidade de Caxias do Sul. 


\section{CONSIDERAÇÕES FINAIS}

Mudanças no fazer implicam mudanças no ser já que ambos estão acoplados (Maturana, 1999). Mas o fazer para mudar o ser precisa estar relacionado a operações de metacognição e de consciência dos processos de fazer, para que possa operar transformações. É nesse sentido que acreditamos que as estratégias e intervenções propostas podem constituir operações que levem às mudanças necessárias para que o processo educativo promova as transformações, tanto nos estudantes como nos professores, desde que estejam envolvidos de forma que se considerem, de fato, participantes nesse processo.

Os resultados das práticas pedagógicas acima descritas revelam a riqueza dessa forma de conceber os processos de ensino, de aprendizagem e de avaliação. Sem dúvida, a implementação de uma metodologia de análise e interpretação de dados coletados, poderá ampliar a compreensão do processo de aprendizagem e a construção de estratégias de intervenção pedagógica compatíveis com o desenvolvimento das competências referidas pelas novas DCNs.

Ao integrar ambientes de aprendizagem ativa, conforme descrevemos, o estudante pode desenvolver autonomia, raciocínio organizado, autocrítica, habilidades de interpretação de textos, de elaboração de ideias e de análise, de trabalho em equipe, dentre outras competências. Além disso, em atividades que promovam a tomada de consciência do que ele pode realizar e desenvolver como competências de valor, o processo de aprender sai do foco diretivo e informativo, para um transformador e criador, mais compatível com as exigências atuais. Entende-se ser mais importante promover aprendizagem significativa do que, apenas, aprovações ou reprovações!

Podemos dizer que, a partir de experiências como essas, os estudantes começam a mudar a concepção de aprender. Só o fato de estarem lendo, interpretando, refletindo, fazendo perguntas, já é um avanço, ainda que pequeno, em relação à maneira tradicional. Não consideramos, portanto, questionamentos ou respostas padronizadas. Procuramos respeitar os diferentes níveis dos estudantes e orientá-los de acordo com o que oferecem como possibilidades. Cada conquista traduz um entendimento a mais e alimenta o passo seguinte. A motivação é, segundo Maturana (1999), impulsionada pelo motor afetivo, de tal forma que o sujeito esteja disposto a buscar respostas a suas dúvidas e seja capaz de construir conhecimentos a partir dos que possui.

\section{REFERÊNCIAS}

ANGELO, T. A.; CROSS, K. P. Classroom assessment techniques: a handbook for college teachers. 2 ed. San Francisco: Jossey-Bass, 1993.

\section{AUSUBEL, D. P. Aquisição e retenção de conhecimentos: uma perspectiva} cognitiva. Lisboa: Plátano, v. 1, 2003.

BRASIL. Letramento Matemático. Brasília. 2010. Disponível em: http://download.inep.gov.br/download/internacional/pisa/2010/letramento_matematico.pdf. Acesso em 16 mai. 2021.

Supremo Tribunal Federal. A garantia da dignidade da pessoa humana é o princípio mais importante na Constituição de 1988, afirma ministra Cármen Lúcia. Brasília, 2018. Disponível em: <http://www.stf.jus.br/portal/cms/verNoticiaDetalhe.asp?idConteudo=386320>. Acesso em 05 set. 2020. 
Ministério da Educação. Diretrizes Curriculares Nacionais para os Cursos de Engenharia. Resolução CNE/CES no 2, de 24 de abril de 2019 - Institui as Diretrizes Curriculares Nacionais do Curso de Graduação em Engenharia. 2019. Disponível em <http://www.in.gov.br/web/dou/-/resolu\%C3\%87\%C3\%83o-n\%C2\%BA-2-de-24-de-abrilde-2019-85344528>. Acesso em: 31 maio 2019.

CID, M. Avaliar para incluir e melhorar as aprendizagens: práticas, obstáculos e possibilidades, Repositório Universidade de Évora, 2017. Disponível em:

<https://dspace.uevora.pt/rdpc/bitstream/10174/22848/1/AVALIAR\%20PARA\%20INCLUIR \%20E\%20MELHORAR\%20AS\%20APRENDIZAGENS.\%20PR\%C3\%81TICAS\%2C\%200 BST\%C3\%81CULOS\%20E\%20POSSIBILIDADES.pdf> Acesso em: 15 abr. 2021.

COLL, C. Aprendizagem escolar e construção do conhecimento. Porto Alegre: Grupo Artes Médicas, 1994.

DE LIMA, P. V. P., et al. Brasil no Pisa (2003-2018): Reflexões no campo da Matemática. Tangram - Revista de Educação Matemática. Dourados, v.3, n.2, p. 03-26, 2020.

ELMÔR-FILHO, G; SAUER, L. Z.; ALMEIDA, N. N.; VILLAS-BOAS, V. Uma Nova Sala de Aula é Possível: Aprendizagem Ativa na Educação em Engenharia, 1. ed. Rio de Janeiro: LTC, 2019.

FREIRE, Paulo. Educação como Prática da Liberdade. Rio de Janeiro: Editora Civilização Brasileira SA. 1967.

GUERRA, M. S. Uma seta no alvo. A avaliação como aprendizagem. Porto: ASA, 2003.

HADJI, C. A Avaliação, Regras do Jogo. Das Intenções aos Instrumentos. Porto: Porto Editora, 1994.

INEP. Instituto Nacional de Estudos e Pesquisas Educacionais Anísio Teixeira. Diretoria de Avaliação da Educação Básica. Relatório Brasil no Pisa 2018 (versão preliminar). Brasília, 2019. Disponível em:

https://download.inep.gov.br/publicacoes/institucionais/avaliacoes_e_exames_da_educac ao_basica/relatorio_brasil_no_pisa_2018.pdf. Acesso em 16 mai. 2021.

\section{LIMA, I. G. A equilibração dos processos cognitivos na aprendizagem de} matemática no ambiente do Mecam. 2004, 220f. Tese (Doutorado em Informática em Educação) - PGIE, UFRGS, Porto Alegre, 2004.

LIMA, I. G.; SAUER, L. Z.; SOARES, E. M. S. O processo de avaliação integrando o processo de ensino aprendizagem por competências na educação em engenharia. In: XXXV Congresso Brasileiro de Educação em Engenharia, COBENGE 2007, Curitiba, Paraná, Brasil.

MORAES, R. Aprender ciências: reconstruindo e ampliando saberes. In: GALIAZZI, M. C., AUTH, M., MORAES, R. MANCUSO, R. (Org.). Construção curricular em rede na educação em ciência: uma aposta de pesquisa na sala de aula. ljuí: Ed. Unijuí, 2007. 
POLYA, G. A arte de resolver problemas. Rio de Janeiro: Interciência, 1977.

PERRENOUD, P. Avaliação: da excelência à regulação das aprendizagens, entre duas lógicas. Porto Alegre, Artmed, 1999.

PIAGET, J. Fazer e compreender. São Paulo: Melhoramentos/EDUSP, 1978.

SAUER, L.Z. O diálogo matemático e o processo de tomada de consciência da aprendizagem em ambientes telemáticos. 2004, 195f. Tese (Doutorado em Informática em Educação) - PGIE, UFRGS, Porto Alegre, 2004.

SCHWARTZ, C. An Academic Adventure. 1991 Disponível em:

<https://www.ocf.berkeley.edu/ schwrtz/Minute.html>. Acesso em: 11 abr. 2021.

SOARES, E. M. S; LIMA, I. G.; SAUER, L. Z. Estratégias de aprendizagem ativa em matemática para educação em engenharia. In: XXXVI Congresso Brasileiro de Educação em Engenharia, COBENGE 2008, São Paulo, Brasil.

SOARES, E. M. S. Comportamentos matemáticos e o ensino de matemática para cursos de engenharia. 1997. 251 f. Tese (Doutorado em Metodologia do Ensino Superior) - Faculdade de Educação, Universidade Federal de São Carlos, São Carlos, 1997.

VILLAS-BOAS, V. et al. Aprendizagem Ativa na Educação em Engenharia. In: Vanderli Fava de Oliveira, Zacarias Chamberlain, Adriano Péres, Paulo Roberto Brandt, Simone Leal Schwertl. (Org.). DESAFIOS DA EDUCAÇÃO EM ENGENHARIA: Vocação, Formação, Exercício Profissional, Experiências Metodológicas e Proposições. 1ed.Blumenau: EdiFURB, 2012, v. 1, p. 59-112.

\title{
THE DELICATE TASK OF ASSESSING LEARNING IN TRAINING BY COMPETENCES IN STEM
}

\begin{abstract}
In this paper, we take as a starting point the effectiveness, already proven, of the use of active learning strategies and methods. In fact, we also understand that this is a methodological alternative to cope, not only to overcome difficulties related to learning, but also to meet expectations regarding the profile and skills that our students need to develop. Given these considerations, we justify, below, our conceptions about the teaching, learning and assessment processes and present some possibilities already identified, of active learning and assessment strategies, which can promote the development of competences in the context of the new DCNs.
\end{abstract}

Keywords: Assessment, Competences, STEM, Engineering education, DCNs 